\title{
Factors associated with bite force in people with Parkinson's disease
}

Taysa Vannoska de Almeida Silva ${ }^{1}$ https://orcid.org/0000-0003-0517-2410

Amanda do Vale Sobral ${ }^{2}$ https://orcid.org/0000-0002-4538-1172

Hilton Justino da Silva ${ }^{3}$ https://orcid.org/0000-0002-6852-3233

Maria das Graças Wanderley de Sales Coriolano ${ }^{1}$ https://orcid.org/0000-0002-7937-7761

Carla Cabral dos Santos Accioly Lins ${ }^{1}$ https://orcid.org/0000-0002-1018-5331
Universidade Federal de Pernambuco UFPE, Programa de Pós-Graduação em Gerontologia, Recife, Pernambuco, Brasil.

2 Universidade Federal de Pernambuco UFPE, Curso de Graduação em Odontologia, Recife, Pernambuco, Brasil. ${ }^{3}$ Universidade Federal de Pernambuco UFPE, Departamento de Fonoaudiologia, Recife, Pernambuco, Brasil.

Research support source: Coordenação de Aperfeiçoamento de Pessoal de Nível Superior - Brasil (CAPES) - Finance Code 001.

Conflict of interests: Nonexistent

\section{(c) (i)}

Received on: September 2, 2021 Accepted on: October 14, 2021

Corresponding address:

Taysa Vannoska de Almeida Silva.

$2^{\mathrm{a}}$ Travessa PIO XII, 58 - Cajueiro Seco

CEP: $54330-323$ - Jaboatão dos

Guararapes, Pernambuco, Brasil

E-mail: taysavnk.fisio@gmail.com

\section{ABSTRACT}

Purpose: to assess the bite force in people with Parkinson's disease and correlate it with age, sex, stage and time of the disease, dentures use, presence of temporomandibular disorder, side of symptom onset, and masticatory preference.

Methods: the Research Diagnostic Criteria for Temporomandibular Disorders and the Hoehn \& Yahr Parkinson's disease scale were used, and the participants' oral cavity was clinically examined. Then, the bite force was measured with an analog dynamometer, whose bar was adapted for mouth grip. The bite force results and their correlation with the variables were analyzed with the independent t-test $(p<0.05)$.

Results: the sample comprised 42 parkinsonians at a mean age of 64 years, $67 \%$ of whom were males. No difference was observed in the correlation with age, temporomandibular disorder, stage and time of Parkinson's disease; or between the sides, symptom onset and masticatory preference. However, there was a significant association between the sexes (males and females) for both sides (right $-p=0.002$; left $p=0.04)$ and denture use for bite force on the right side $(p=0.03)$.

Conclusion: being a female and using dentures are factors associated with decreased bite force in people with Parkinson's disease.

Keywords: Parkinson Disease; Bite Force; Masticatory Muscles 


\section{INTRODUCTION}

Parkinson's disease (PD) is a chronic degenerative disease of the central nervous system (CNS), characterized by the degeneration of the compact portion of the substantia nigra in the midbrain ${ }^{1}$. It causes the loss of dopaminergic neurons and the appearance of the main motor symptoms: bradykinesia, tremor at rest, stiffness, and postural and gait instability ${ }^{2}$.

It is the second neurodegenerative disease with the highest incidence in older adults, although its etiology is unknown ${ }^{3}$. It more commonly affects men, and its onset usually takes place at 60 to 80 years old, increasing in incidence with age ${ }^{4,5}$.

The motor symptoms may cause orofacial manifestations, such as inexpressive facial appearance ("masked face"), tremors and stiffness in the forehead, eyelids, lips, and tongue muscles, and pharyngeal motor deficit ${ }^{6}$. These changes may cause facial and temporomandibular joint pain and dental traumas ${ }^{7}$. Hence, the person may present temporomandibular disorder (TMD), a need for dentures, and impaired mastication and swallowing ${ }^{6,8,9}$.

Mastication is a movement coordinated and regulated by the CNS to fragment food and prepare it for swallowing ${ }^{10}$. The literature shows that the maximum bite force (BF) and muscle mass decrease with age, increasing the number of masticatory cycles necessary to break and swallow the food ${ }^{11}$. Since the CNS is responsible for making the necessary orofacial changes to set the masticatory rhythm, neurological disorders such as PD can impair the masticatory muscle activity ${ }^{6,12,13}$.

One way to assess mastication is by observing the force exerted by the masticatory muscles to break the food, which is called BF. It can be measured with a unilateral and/or bilateral force transducer - which has a sensor that measures tension and compression forces in newtons $(\mathrm{N})$ - or with a dynamometer ${ }^{14}$ which measures the BF in kilograms-force (kgf) or $\mathrm{N}$. They are placed between the upper and lower dental arches in the oral cavity to pick up the muscle action of the mandibular elevators ${ }^{15,16}$.

Thus, this study aimed at assessing BF and correlating it with age, sex, stage and time of $\mathrm{PD}$, presence of TMD, use of dentures, side of symptom onset, and masticatory preference in PD patients attended at a public university hospital.

\section{METHODS}

This is an analytical cross-sectional study, approved by the Human Research Ethics Committee of the Center for the Health Sciences at the Universidade Federal de Pernambuco (Federal University of Pernambuco UFPE), Brazil, under evaluation report no. 1.763.512. It was developed between November 2016 and November 2017 at the Neurology Outpatient Center of the Clinics Hospital $(\mathrm{HC})$, in partnership with the UFPE Pro-Parkinson public outreach program.

The participants were selected by convenience from a nonprobabilistic sample, based on the number of people who attended the HC/UFPE Pro-Parkinson public outreach program in 2016. Those who voluntarily agreed to participate were informed of the objectives of the research and signed the informed consent form. They were selected based on the following eligibility criteria: patients with a clinical diagnosis of idiopathic PD in the mild (1 and 2) and moderate stages (3) of the disease, established by the worldwide used Hoehn \& Yahr classification scale (1967) ${ }^{17}$; in the "on" phase (under the effects of the antiparkinsonian drug); having the posterior teeth or using complete or partial removable dentures; with or without TMD; undergoing speech-language-hearing follow-up; autonomous and/or being cared for by relatives. People with other neurological diseases associated with PD; with cognitive impairment according to the Mini-Mental State Examination (MMSE); with toothless upper and lower dental arches; or having been submitted to some type of mandibular or neurological surgery due to PD were excluded.

The volunteers were interviewed in person by a multiprofessional team made up of a physician, dental surgeon, and physical therapist to assess the inclusion and exclusion criteria. The MMSE was used to assess cognitive function. It is an instrument with 11 items and a maximum score of 30 points, which takes 5 to 10 minutes to apply. Its cutoff scores depend on the person's educational level - illiterates: 13 points; low educational level: 18 points; 8 or more years at school: 26 points $^{18}$.

Then, the TMD assessment instrument (Research Diagnostic Criteria for Temporomandibular Disorders - RDC/TMD) was applied. Its approach has two main axes that enable the measurement of physical findings with facial and oral cavity clinical examination (Axis I) and the assessment of sociodemographic data and psychosocial status (Axis II). The data obtained in Axis II are the basis for the diagnoses addressed in Axis 
1 $^{19}$. The RDC/TMD sociodemographic questionnaire furnished the data on age (in full years, based on the date of birth and of data collection) and sex (male or female).

Besides these data, two self-reported questions were asked: 1- "What was the side of PD symptom onset (SSO)?"; and 2- Do you have a preferred side of mastication?". The dental surgeon assessed whether they used dentures and their type and inspected the participants' oral cavity, not considering for this study either the type of occlusion or dental changes. Then, the force record protocol was performed.

The BF was obtained with an analog dynamometer, model sForce DPM NM (Miotec Biomedical Equipment Ltda., Porto Alegre, Brazil). It has a bar adapted for oral use, with a capacity of $100 \mathrm{kgf}$, connected to the equipment Miotool Face 200/400 (Miotec Biomedical Equipment Ltda., Porto Alegre, Brazil) via USB. The dynamometer was wrapped with plastic wrap and positioned only in the right and left first molar region, recording the data alternately between the sides.

Initially, the volunteers performed the test to standardize the data with the maximum voluntary contraction (MVC) of the masticatory muscles, having cotton rolls placed between the dental arches in the molar region. Then, the protocol was performed ${ }^{20}$, which consisted of biting the analog dynamometer for 5 seconds, with 10-second rest intervals in between actions, which were repeated three times ${ }^{20}$. The maximum $B F$ values of each test were used to obtain the mean value. The data were analyzed with the MiotecSuit 1.0 software, which belongs to the Oral-Motor Function Laboratory of the originating institution.

The data were then tabulated and compiled with descriptive analysis and measures of central tendency and dispersion. The Shapiro-Wilk test was used to assess the normality of the sample; as the normality was verified, the independent t-test was used to assess the BF between both sides with the variables studied. The SPSS 22.0 statistical program was used, considering $p<0.05$.

\section{RESULTS}

A total of 170 people were recruited for this study, of whom 128 were excluded because they did not meet the eligibility criteria. Hence, the final sample comprised 42 subjects.

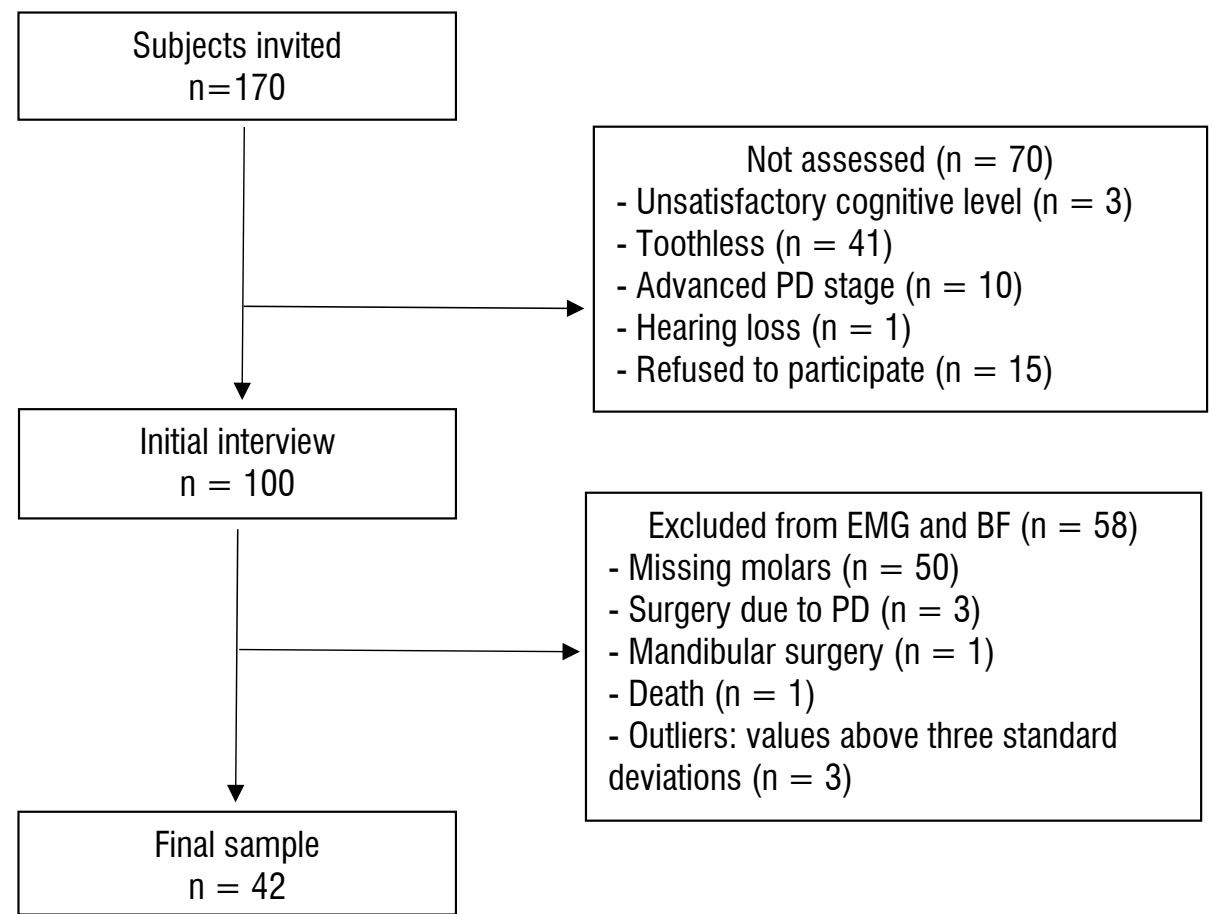

Figure 1. Flowchart of the sample selection 
There was a predominance of males (64\%), at the mean age of $64( \pm 9)$ years, ranging from 43 to 80 years. The mean time of diagnosis was $7( \pm 4)$ years, and the highest percentage $(79 \%)$ were in the mild stage of PD. The symptom onset self-report indicated a higher incidence on the left side $(60 \%)$ and mostly a preference to use the right side for mastication (43\%). Also, $69 \%$ of the sample used dentures, and most of them did not have TMD (71\%).

Table 1. Distribution of the sociodemographic variables, time and stage of the disease, denture use, temporomandibular disorder, side of symptom onset, and masticatory preference. Recife, Brazil, 2017

\begin{tabular}{lcc}
\hline Variables & $\mathbf{n}(\%)$ & $\overline{\mathbf{X}}( \pm \mathbf{)}$ \\
\hline Age (years) & $30(71 \%)$ & \\
$\geq 60$ years & $12(28 \%)$ & \\
$<60$ years & $27(64 \%)$ & $7 \pm 4$ \\
Sex & $15(36 \%)$ & \\
Males & & \\
Females & $19(45 \%)$ \\
Time of PD diagnosis (years) & $23(55 \%)$ \\
$2 \mid--5$ years & & \\
$6 \mid--20$ years & $33(79 \%)$ \\
Stages of PD & $9(21 \%)$ \\
Mild Stage & & \\
Moderate Stage & $29(69 \%)$ \\
Denture Use & $13(31 \%)$ \\
Yes & $12(29 \%)$ \\
No & $30(71 \%)$ \\
TMD & \\
Yes & $17(40 \%)$ \\
No & $25(60 \%)$ \\
Side of PD symptom onset & \\
Right side & $18(43 \%)$ \\
Left side & $14(33 \%)$ \\
Masticatory Preference & $10(24 \%)$ \\
Right side & & \\
Both sides of the dental arch & & \\
Left side & & \\
\hline
\end{tabular}

Captions: PD: Parkinson's disease; TMD: temporomandibular disorder; \%: percentage; $\bar{X}$ : mean; \pm : standard deviation.

The minimum, maximum, and mean values of right and left BF are shown in Table 2. Applying the independent t-test, no significant difference was observed between the two sides of the dental arch, the SSO, and the preferred side of mastication. 
Table 2. Bite force values (in kgf) expressed in maximum, mean, and minimum value

\begin{tabular}{lccc}
\hline & \multicolumn{2}{c}{ Bite Force } & \multirow{2}{*}{ P-value $^{*}$} \\
\cline { 2 - 3 } & Right & Left & \\
Variation (minimum - maximum) & $3-57$ & $5-53$ & 0.45 \\
Mean $( \pm)$ & $25(16)$ & $21(13)$ & \\
Symptom onset & & & 0.31 \\
Right & $26(15)$ & $21(13)$ & 0.48 \\
Left & $24(17)$ & $21(14)$ & \\
Masticatory Preference & & & 0.62 \\
Right & $23(14)$ & $21(12)$ & 0.29 \\
Left & $22(18)$ & $15(12)$ & 0.54 \\
Both sides & $30(17)$ & $26(15)$ & \\
\hline
\end{tabular}

Captions: kgf: kilogram-force; $\bar{X}$ : mean; \pm : standard deviation; independent t-test; $p<0.05^{\star}$.

The analysis between BF and sex, age, stage of PD, time of diagnosis, denture use, and presence of TMD revealed a significant association between males and females for both sides (right BF - $p=0.002$; left BF $p=0.04)$ and denture use for right side $B F(p=0.03)$

Table 3. Values (in kgf) expressed in mean and standard deviation found in the factors associated with bite force on the right and left sides

\begin{tabular}{|c|c|c|c|}
\hline Age & $<60$ years $(n=12)$ & $\geq 60$ years $(n=30)$ & P-value \\
\hline Right BF & $28(15)$ & $24(17)$ & 0.54 \\
\hline Left BF & $26(14)$ & $19(13)$ & 0.15 \\
\hline Sex & Males $(n=27)$ & Females $(n=15)$ & p \\
\hline Right BF & $30(16)$ & $23(15)$ & $0.002^{*}$ \\
\hline Left BF & $16(10)$ & $16(8)$ & $0.04^{*}$ \\
\hline Stage of PD & Mild Stage $(n=33)$ & Moderate Stage $(n=9)$ & $\mathrm{p}$ \\
\hline Right BF & $25(15)$ & $28(21)$ & 0.61 \\
\hline Left BF & $20(12)$ & $28(17)$ & 0.11 \\
\hline Time of PD diagnosis & $2 \mid-5(n=19)$ & $6 \mid-20(n=23)$ & p \\
\hline Right BF & $22(15)$ & $28(16)$ & 0.20 \\
\hline Left BF & $20(14)$ & $22(13)$ & 0.61 \\
\hline Dentures use & With Dentures $(n=29)$ & Without Dentures $(n=13)$ & p \\
\hline Right BF & $20(14)$ & $31(15)$ & $0.03^{*}$ \\
\hline Left BF & $20(15)$ & $23(9)$ & 0.07 \\
\hline TMD & With TMD $(n=12)$ & Without TMD $(n=30)$ & $p$ \\
\hline Right BF & $19(13)$ & $24(15)$ & 0.63 \\
\hline Left BF & $20(13)$ & $21(14)$ & 0.78 \\
\hline
\end{tabular}

Captions: BF: bite force; PD: Parkinson's disease; BF: bite force; TMD: temporomandibular disorder; kgf: kilogram-force; $\bar{X}$ : mean; \pm : standard deviation; independent t-test; ${ }^{*} p(<0.05)$. 


\section{DISCUSSION}

Previous research analyzing the sociodemographic profile of people with PD observed data that corroborate those found in this study regarding mean age above 60 years, higher frequency of PD in men, mean of 7 years of PD diagnosis, and the greater number of people in the mild stage ${ }^{21-23}$.

Anatomical and physiological characteristics - such as sex, height, weight, dental status, facial morphology, age, denture use, presence of TMD, and masticatory preference - influence the direct BF measures ${ }^{6,15,24-26}$. However, no correlation between BF and PD stage, SSO, and time of diagnosis was found in the literature researched to compare with this study.

Physiological changes, such as stiffness and bradykinesia, present in the orofacial muscles of people with $\mathrm{PD}$, lead to compensations in the structures involved in mastication ${ }^{27}$. The occlusal instabilities and interferences may overload the stomatognathic system and alter the muscle coordination between the two sides ${ }^{28}$. This aspect was observed in the mean BF value, which was higher on the right than on the left side, characterizing an asymmetry between the sides. This corroborates research that demonstrated an existing asymmetry in masticatory muscle activity in people with normal teeth and without pathologies ${ }^{29,30}$.

The asymmetry in the motor symptoms lasts throughout the disease. Hence, the SSO is more impaired than its contralateral - which is eventually affected, though to a lower degree. The impairment on the SSO may negatively influence motor skill performance $^{31}$. The association between BF and SSO showed that the left side, which had the highest percentage of symptom onset, had higher mean BF values on the contralateral side. However, no articles were found in the literature researched correlating these data for discussion.

To properly handle and transport food, the mastication counts with support from synchronous muscle activity and uniform force, with ample, bilateral, and alternated masticatory cycles ${ }^{25}$. Hence, the masticatory force is distributed so as to synchronize and balance the musculature of the stomatognathic system and its functions ${ }^{32}$. When the person prefers to masticate more on one side of the mouth than the other, muscle changes take place, with greater power on the side most often used, whereas the musculature on the other side is elongated and has less tonus ${ }^{32}$. Khan et al. ${ }^{33}$ studied 95 people without PD and observed that the dominant masticatory side has a higher mean BF value than the nondominant side. Such an aspect was observed in this study, whose mean BF values were higher on the right side in both those who reported a masticatory preference on one side of the dental arch and who had bilateral mastication.

Studies have demonstrated that age and sex are important factors that influence the resistance of the mandibular elevator muscles regarding BF, with a difference between the sexes and remaining constant between 20 and 50 years of age ${ }^{11,24}$. Takaki et al. ${ }^{24}$ analyzed the maximum BF in 100 people, divided into five groups by age and sex. They observed that, regardless of age, the BF was always greater in men than in women. That corroborates with the result in this research, which observed a significant decrease in $\mathrm{BF}$ values between males and females on both sides of the dental arch. Palinkas et al. ${ }^{11}$ verified that the increase in age reduces the force and thickness of the masseter and temporal muscles. This agrees with the lower mean $\mathrm{BF}$ value found in the older participants in this research, in relation to the younger ones.

Ribeiro et al. ${ }^{9}$ analyzed the masticatory function in people with $\mathrm{PD}$ and found a mean time of diagnosis similar to the one observed in this research. However, they did not associate this information with BF. The BF analysis did not show an association between the patients' different times of diagnosis, and no studies were found in the literature with a similar association for discussion. Concerning the stage of the disease, the BF increased as the signs and symptoms advanced, differently from the study by Bakke et al. ${ }^{34}$. They assessed the mastication in people with PD and observed that the orofacial function may be impaired in the moderate and advanced stages of the disease. They also pointed out that as the motor symptoms progressed, the orofacial and dental problems grew more intense. However, the finding of this research could be correlated with a postural abnormality. Tinazzi et al. ${ }^{35}$ studied postural abnormalities in PD and observed that they are associated with males, aging, longer time of disease onset, advanced stage, and a bradykinetic/rigid phenotype. In this study, the basic forms of PD presentation (tremoring and rigid/akinetic) were not measured.

Silva et al. ${ }^{8}$ assessed the prevalence of TMD in 110 people with PD and obtained a prevalence of $35 \%$ in this population, which corroborates what has been observed in the present research. The BF values obtained in the TMD group were lower than in the non-TMD group, which was also observed in the study by Todic et al. ${ }^{26}$. They associated the presence 
of TMD with $\mathrm{BF}$ in people without PD and found that the presence of the disorder reduces the $\mathrm{BF}$ values and that signs present in TMD (such as muscle pain, temporomandibular joint pain, limited mouth opening, and crepitations) can affect BF. However, no articles were found in the literature researched correlating BF, $P D$, and TMD to better discuss the findings.

Also, studies ${ }^{6,36}$ demonstrated that the changes in the stomatognathic system in PD increase the incidence of denture use in this population, which was also observed in this study. People with natural teeth have higher BF values, with a significant difference on the right side of the dental arch. Dentures users are known to have impaired masticatory function, including maximum voluntary BF and muscle effort when biting and masticating ${ }^{36}$. Also, Schimmel et al. ${ }^{37}$ assessed BF and surface electromyography in toothless older adults who used complete or partial dentures and observed that denture instability probably prevents denture users to use their mandibular muscle full potential, especially in unilateral bite and mastication.

The present study had the limitations characteristic of cross-sectional design, which only enables associations, not making it possible to reach causality conclusions. Another limitation was because the participants did not have the lower molar teeth to use in BF, limiting the sample size. Also, there was a scarcity of publications on this topic in people with PD to broaden and discuss the outcomes found in this study. Thus, longitudinal studies must be carried out to establish the cause-and-effect relationships of the variables analyzed. These investigations can provide valuable information on the prevention and treatment strategies for this population.

\section{CONCLUSION}

This study revealed the presence of BF asymmetry between the two sides of the dental arch. Its association with age, sex, stage and time of PD, presence of TMD, dentures use, SSO, and masticatory preference demonstrated that being a female and using removable dentures decreased the BF in the population studied.

\section{ACKNOWLEDGMENTS}

We would like to express our deepest gratitude to Neurologist Dr. Amdore Asano for granting access to the Neurology Outpatient Center of the Clinics Hospital, which made data collection possible, and to Dr. Nadja Asano, for the technical support during the activities carried out at the outpatient center. This study was financed in part by the Coordenação de Aperfeiçoamento de Pessoal de Nível Superior - Brasil (CAPES) - Finance Code 001.

\section{REFERENCES}

1. Cabreira V, Massano J. Doença de Parkinson: revisão clínica e atualização. Acta Med Port. 2019;32(10):661.

2. Rodríguez-Violante M, Zerón-Martínez R, CervantesArriaga A, Corona T. Who can diagnose Parkinson's disease first? Role of pre-motor symptoms. Arch Med Res. 2017;48(3):221-7.

3. Tysnes O-B, Storstein A. Epidemiology of Parkinson's disease. J Neural Transm. 2017;124(8):901-5.

4. Ramazzina I, Bernazzoli B, Costantino C. Systematic review on strength training in Parkinson's disease: an unsolved question. Clin Interv Aging [journal on the internet] 2017 [accessed 2021 Aug 2];12:619-28. Available at: https://pubmed.ncbi. nlm.nih.gov/23087283/

5. Hirsch L, Jette N, Frolkis A, Steeves T, Pringsheim T. The Incidence of Parkinson's disease: a systematic review and meta-analysis. Neuroepidemiology. 2016;46(4):292-300.

6. Massimo C, Biagio R, Giovanni C, Massimo C, Pasquale $S$, Andrea DG et al. Orofacial functions and chewing efficiency in elderly patients with Parkinson's disease rehabilitated with removable prostheses. Open Dent J. 2020;14(1):13-8.

7. Friedlander $\mathrm{AH}$, Mahler $\mathrm{M}$, Norman $\mathrm{KM}$, Ettinger RL. Parkinson disease: systemic and orofacial manifestations, medical and dental management. J Am Dent Assoc. 2009;140(6):658-69.

8. Silva RM da, Santos V, Silva TV de A, Lins CC dos SA. Prevalence of temporomandibular joint disorder in people with Parkinson's disease in a public university hospital. Rev. CEFAC. 2019;21(3):1-10.

9. Ribeiro GR, Campos $\mathrm{CH}$, Rodrigues Garcia RCM. Parkinson's disease impairs masticatory function. Clin Oral Investig. 2017;21(4):1149-56.

10. Peyron MA, Woda A, Bourdiol P, Hennequin M. Age-related changes in mastication. J Oral Rehabil. 2017;44(4):299-312.

11. Palinkas M, Nassar MSP, Cecílio FA, Siéssere $S$, Semprini M, Machado-De-Sousa JP et al. Age and gender influence on maximal bite force and masticatory muscles thickness. Arch Oral Biol. 2010;55(10):797-802. 
12. Quintero A, Ichesco E, Schutt R, Myers C, Peltier S, Gerstner GE. Functional connectivity of human chewing. J Dent Res. 2013;92(3):272-8.

13. Verri ED, da Silva GP, Fioco EM, Silva NS, Fabrin SCV, Zanella CAB et al. Effects of Parkinson's disease on molar bite force, electromyographic activity and muscle thickness of the masseter, temporal and sternocleidomastoid muscles: a case-control study. J Oral Rehabil. 2019;46(10):912-9.

14. Araújo sCCS de, Vieira MM, Gasparotto CA, Bommarito $S$. Bite force analysis in different types of angle malocclusions. Rev. CEFAC. 2014;16(5):1567-78.

15. Melo DG de, Bianchini EMG. Relationship between electrical activity of the temporal and masseter muscles, bite force, and morphological facial index. CoDAS. 2016;28(4):409-16.

16. Varga S, Spalj S, Lapter Varga M, Anic Milosevic S, Mestrovic S, Slaj M. Maximum voluntary molar bite force in subjects with normal occlusion. Eur J Orthod. 2011;33(4):427-33.

17. Hoehn MM, Yahr MD. Parkinsonism: onset, progression and mortality. Neurology. 1967;17(5):427-42.

18. Bertolucci P, Brucki S, Campacci S. The Mini-Mental State Examinationin a general population: impact of educational status. Arq Neuropsiquiatr. 1994;52(1):1-7.

19. Lucena LBS de, Kosminsky M, Costa LJ da, Góes PSA de. Validation of the Portuguese version of the RDC/TMD Axis II questionnaire. Braz Oral Res. 2006;20(4):312-7.

20. Nascimento G, Lima L, Rodrigues C, Cunha R, Cunha $D$, Silva $H$. Verification of bite force and the electrical activity of masseter muscle during chewing in laryngectomized. Rev Bras Odontol. 2011;68(2):175-9.

21. Spitz M, Tinoco V, Menezes F, Pereira J. Analysis of motor symptoms in patients with Parkinson's disease from a tertiary hospital in Rio de Janeiro. Rev Bras Neurol. 2017;53(3):14-8.

22. Coriolano M, Silva E, Fortuna E, Asano A, Monteiro $D$, Lins O. Epidemiological profile of patients with Parkinson's disease (PD) in the Clinical Hospital of Federal University of Pernambuco. Neurobiologia. 2013;76(1):1-2.

23. Leandro LA, Teive HAG. Fatores associados ao desempenho funcional de idosos portadores da doença de Parkinson. Rev Kairós Gerontol. 2017;20(2):161-78.

24. Takaki $P$, Vieira M, Bommarito S. Maximum bite force analysis in different age groups. Int. Arch. Otorhinolaryngol. 2014;18(3):272-6.

25. Gomes SGF, Custodio W, Faot F, Cury AADB, Garcia RCMR. Chewing side, bite force symmetry, and occlusal contact area of subjects with different facial vertical patterns. Braz Oral Res. 2011;25(5):446-52.

26. Todic J, Martinovic B, Pavlovic J, Tabakovic S, Staletovic M. Assessment of the impact of temporomandibular disorders on maximum bite force. Biomed Pap. 2019;163(3):274-8.

27. Albuquerque LCA, Silva HJ da. Jaw movement in people with Parkinson's Disease. CoDAS. 2016;28(2):193-6.

28. Chiodelli L, Pacheco A de B, Missau TS, Silva AMT da, Corrêa ECR. Association among stomatognathic functions, dental occlusion and temporomandibular disorders signs in asymptomatic women. Rev. CEFAC. 2015;17(1):117-25.

29. Mapelli A, Zanandréa Machado BC, Giglio LD, Sforza C, De Felício CM. Reorganization of muscle activity in patients with chronic temporomandibular disorders. Arch Oral Biol. 2016;72:164-71.

30. Nalamliang $N$, Sumonsiri $P$, Thongudomporn U. Are occlusal contact area asymmetry and masticatory muscle activity asymmetry related in adults with normal dentition? CRANIO ${ }^{\circledR}$ [journal on the internet] 2020 [accessed 2021 Aug 2]; 13;1-9. Available at: https://www.tandfonline.com/doi/abs/10.1080/0 8869634.2020.1764270?journalCode =ycra20

31. Lahr J, Pereira MP, Pelicioni PHS, Batistela RA, Gobbi LTB. The onset side of the disease influences the manual dexterity in patients with Parkinson's disease. Rev Ter Ocup da Univ São Paulo. 2018;29(3):223-9.

32. Nascimento GKBO, Lima LM de, Freitas MCR de, Silva EGF da, Balata PMM, Cunha DA da et al. Preference side masticatory and facial symmetry in total laryngectomy: clinical and electromyographic study. Rev. CEFAC. 2013;15(6):1525-32.

33. Khan SIR, Rao D, Ramachandran A, Ashok BV. Comparison of bite force on the dominant and nondominant sides of patients with habitual unilateral chewing: a pilot study. Gen Dent. 2020;68(2):60-3.

34. Bakke M, Larsen SL, Lautrup C, Karlsborg M. Orofacial function and oral health in patients 
with Parkinson's disease. Eur $J$ Oral Sci. 2011;119(1):27-32.

35. Tinazzi M, Gandolfi M, Ceravolo R, Capecci M, Andrenelli E, Ceravolo MG et al. Postural abnormalities in Parkinson's disease: An epidemiological and clinical multicenter study. Mov Disord Clin Pract. 2019;6(7):576-85.

36. Ribeiro GR, Campos CH, Rodrigues Garcia RCM. Influence of a removable prosthesis on oral health-related quality of life and mastication in elders with Parkinson disease. J Prosthet Dent. 2017;118(5):637-42.

37. Schimmel M, Memedi K, Parga T, Katsoulis J, Müller F. Masticatory performance and maximum bite and lip force depend on the type of prosthesis. Int J Prosthodont. 2017;30(6):565-72. 